\title{
Assessment of Lipiodol Deposition and Residual Cancer for Hepatocellular Carcinoma After Transcatheter Arterial Chemoembolization via Iodine- Based Material Decomposition Images with Spectral Computed Tomography Imaging: A Preliminary Study
}

\author{
Yanhong Xu ${ }^{1}$; An Xiao ${ }^{2}$; Jia Yang ${ }^{1}$; Zaixian Zhang ${ }^{1}$; Guixiang Zhang ${ }^{1,}$ \\ ${ }_{1}^{1}$ Department of Radiology, Shanghai First People's Hospital, School of Medicine, Jiao Tong University, Shanghai, China \\ ${ }^{2}$ Department of Interventional Radiology, Shanghai First People's Hospital, School of Medicine, Jiao Tong University, Shanghai, China \\ ${ }^{*}$ Corresponding author: Guixiang Zhang, Department of Radiology, Shanghai First People’s Hospital, School of Medicine, Jiao Tong University, Shanghai, China. Tel: +86-2163240090, \\ Fax:+86-2163240825, E-mail: guixiangzhang@sina.com
}

Received: December 10, 2014; Revised: January 13, 2015; Accepted: February 14, 2015

\begin{abstract}
Background: It is critical to follow up hepatocellular carcinoma (HCC) after transcatheter arterial chemoembolization (TACE) in clinical practice. Computed tomography (CT) is used to assess lipiodol deposition, whereas it is difficult to assess hypovascular residual cancer masked by lipiodol. In contrast, magnetic resonance imaging (MRI) is superior to CT in showing residual cancer, but cannot display lipiodol deposition.

Objectives: The aim of this study was to investigate the value of spectral CT imaging in both lipiodol deposition and residual cancer for HCC patients after TACE.

Patients and Methods: Ten HCC patients after treated with TACE underwent Discovery CT750 HD and MRI750 3T examination. Receiver operating characteristic (ROC) curves of iodine-based material decomposition images, monochromatic images and conventional CT images were generated.

Results: Consequently,30 residuallesions were detected in MRI of 10 patients. Theywere found in iodine-based images and monochromatic images versus 29 in conventional CT images. The area under ROC curves for the lesion-to-normal parenchyma ratio (LNR) on arterial phase (AP) in iodine-based material decomposition images, monochromatic images and conventional CT images were 0.933 , 0.833 and 0.817 , respectively.

Conclusion: The study data highlighted good value of iodine-based material decomposition images of spectral CT in assessment of both lipiodol deposition and residual cancer for follow-up of HCC patients previously treated with TACE.
\end{abstract}

Keywords: Tomography; Magnetic Resonance Imaging; Carcinoma; Hepatocellular

\section{Background}

Hepatocellular carcinoma (HCC) is primarily an ordinary cause of cancer-related death worldwide, especially in China (1-6). The incidence of HCC keeps the highest in Eastern Asia and Africa, while its frequency has been increased rapidly in western countries and Japan as well $(7,8)$. Transcatheter arterial chemoembolization (TACE) is one of the most common therapeutic methods for large or multinodular HCCs, which cannot undergo other treatments such as resection or radiofrequency ablation (9). After TACE treatment, follow-up imaging modalities such as computed tomography (CT) and magnetic resonance imaging (MRI) are performed to assess therapeutic efficacy such as lipiodol deposition and residual cancer. However, there are some limitations in individuals. For example, a hypovascular residual cancer can be more difficult to assess on CT, because ac- cumulation of lipiodol in tumors can mask its enhancement. In contrast, MRI is superior to CT in showing residual tumor (10-13), but cannot demonstrate lipiodol deposition. Therefore, there is a need for a new option able to monitor both lipiodol deposition and residual cancer for HCC after TACE. Recently, a new CT scanning mode depended on the rapid switching of high and low tube voltages on neighboring views during a single rotation has been generated on high-definition Discovery CT750 HD scanner. This spectral CT imaging model is called Gemstone Spectral Imaging (GSI) mode, which provides correct registration of data sets for conduction of accurate material decomposition images (e.g. waterand iodine-based material decomposition images) for quantitative density measurement and monochromatic images at energy levels ranging from 40 to $140 \mathrm{keV}$ for

Copyright ( ) 2015, Tehran University of Medical Sciences and Iranian Society of Radiology. This is an open-access article distributed under the terms of the Creative Commons Attribution-NonCommercial 4.0 International License (http://creativecommons.org/licenses/by-nc/4.0/) which permits copy and redistribute the material just in noncommercial usages, provided the original work is properly cited. 
optimized angiographic imaging. Spectral CT has potential to make hyperenhancing lesions brighter on the attenuation scale by taking advantage of the markedly increased density of iodine at photon energy levels just above the outmost electron binding energy.

\section{Objectives}

The aim of this study was to investigate the usefulness of spectral CT imaging in assessment of lipiodol deposition and residual cancer for HCC after TACE.

\section{Patients and Methods}

\subsection{Patients}

The ethics committee at our institution approved this prospective study and all patients provided written informed consent. From April to November 2014, 10 HCC male patients (mean age of 61 years, ranged 50 to 73 years) after TACE underwent both MRI and multiplephase CT scanning. The examiner of CT and MRI was identical for 10 cases. None of the cases underwent other treatment options except TACE. TACE sessions were performed for patients from 1 to 6 times. The interval between TACE and MRI imaging examination was 2-3 days, and the interval between MRI and CT imaging examination was 7-14 days.

\subsection{MRI Examinations}

Three-phase contrast material-enhanced MRI examinations were performed using the Discovery MRI750 3T scanner (General Electric Healthcare, Milwaukee, USA) with a peak gradient amplitude of $45 \mathrm{mT} / \mathrm{m}$ and maximal gradient slope of $200 \mathrm{~T} / \mathrm{ms}$. An eight-channel torso phased array coil was centered over the liver. The conventional MRI protocol was adopted in this experiment containing transverse respiratory-navigated T2weighted fat-suppressed turbo spin echo sequence [repetition time (TR)/echo time $(\mathrm{TE})=3500 / 84 \mathrm{~ms}$; section thickness, $5 \mathrm{~mm}$; intersection gap, $1 \mathrm{~mm}$; field of view optimized to patients' body habitus, $285 \times 214-308$ $\times 380 \mathrm{~mm}$; matrix, $168 \times 320$ l and transverse T1-weighted in-phase and out-phase gradient echo [TR/TE $=6.8 / 2.35$ (in-phase), 4.75 (out-phase) ms; section thickness, 5 $\mathrm{mm}$; intersection gap, $1 \mathrm{~mm}$; field of view optimized to patients' body habitus, $285 \times 214-308 \times 380 \mathrm{~mm}$; matrix, $180 \times 320$ ]. Dynamic imaging was performed once before and three times after intravenous administration of gadopentetate dimeglumine (Magnevist; Bayer Healthcare, Germany) with a transverse breath-hold T1weighted three-dimensional volumetric interpolated body examination sequence $(\mathrm{TR} / \mathrm{TE}=5.0 / 2.3 \mathrm{~ms}$; section thickness, $2 \mathrm{~mm}$; no intersection gap; field of view optimized to patients' body habitus, $285 \times 214-308 \times 380$ $\mathrm{mm}$; matrix, $270 \times 360$ ). Gadopentetate dimeglumine was administered at a dose of $0.1 \mathrm{mmol} / \mathrm{kg}$ and a rate of $2 \mathrm{~mL} / \mathrm{s}$ followed by a $20 \mathrm{~mL}$ saline flush by a power injector (Spectris; Medrad, Pittsburgh, PA, USA). The acquisitions were performed at $30 \mathrm{~s}, 60 \mathrm{~s}$ and $180 \mathrm{~s}$ after contrast administration during the hepatic arterial phase (AP), portal venous phase (PP) and delayed phase, respectively.

\subsection{CT Examinations}

Two-phase contrast material-enhanced spectral CT examinations were used by the Discovery CT750 HD scanner (General Electric Healthcare, Wisconsin, USA). All patients were scanned craniocaudally while in the supine position. After scout CT scanning, patients received nonionic contrast materials (ioversol, Optiray 350; Tyco Healthcare, Montreal, Quebec, Canada) with antecubital venous access at a rate of 3-4 mL/s. A total of 70-110 $\mathrm{mL}(1.5 \mathrm{~mL}$ per kilogram of body weight) was injected during phases of AP and PP. AP scanning automatically began at 30 seconds after the trigger attenuation threshold [100 Hounsfield unit (HU)] was reached at the level of supraceliac abdominal aorta (10). PP scanning began with a delay of 30 seconds after AP scanning. AP and PP scanning were performed in the spectral imaging mode with fast tube voltage switching between 80 and $140 \mathrm{keV}$ on adjacent views during a single rotation. Other scanning parameters were as follows; collimation thickness of $0.625 \mathrm{~mm}$, tube current of $600 \mathrm{~mA}$, rotation speed of 0.6 second, helical pitch of 0.983 and CT dose index volume of $21.8 \mathrm{mGy}$ (comparable to $21.5 \mathrm{mGy}$ dose administered for conventional contrast-enhanced liver scanning in a normal-size patient with body mass index within normal range at our institution). CT images were reconstructed by projection-based materialdecomposition software and a standard reconstruction kernel. The adaptive statistical iterative reconstruction algorithm was applied to suppress image noise on decomposition images. Three types of images were reconstructed from the single spectral CT acquisition for analysis: conventional polychromatic images obtained at $140 \mathrm{keV}$, iodine-based material decomposition images and monochromatic images obtained at energies ranging from 40 to $140 \mathrm{keV}$.

\subsection{Imaging Analyses}

MRI imaging studies were evaluated in consensus by two radiologists (each with more than 10 years experience in abdominal imaging) on a picture archiving and communication system (PACS) (Impax 6.4, Agfa, Mortsel, Belgium). The gold standard of a residual cancer was defined as a hypervascular nodule seen on gadoxetic acid-enhanced arterial phase MRI images with a washout pattern considered a residual HCC (14). Washout, a unique feature of HCC, was described as a change from high intense relative to the intense of the liver during the AP to isointense or hypointense relative to that of the liver during the PP. All the measurements of 
XuYetal.

spectral CT were performed on an advanced workstation (AW4.4; GE Healthcare, Waukesha, WI, USA) with the GSI viewer. The default 70-keV monochromatic images and iodine-based material decomposition images were reviewed. Circular or elliptical regions of interest (ROIs) were drawn to encompass as much of the hyperenhancing portion of the lesion as possible (mean pixel number 100, range 20-190) from three contiguous slices. Mean CT data for the adjacent hepatic parenchyma as the background regions was obtained by manually placing circular ROIs in the hepatic parenchyma (mean pixel number of 200, range 100-280). Areas of focal change in hepatic parenchymal attenuation, large vessels and prominent artefacts were avoided carefully. To ensure consistency, all measurements were performed three times at different image levels and average values were calculated. For all measurements, the size, shape and position of the ROIs were kept consistent between the two phases by applying the copy-and-paste function. The spectral CT imaging studies were assessed by other two radiologists, each of them with more than eight years experiences in abdominal imaging. The GSI viewer software package automatically calculated the CT values, iodine (water), water (iodine) concentration for the lesions and normal hepatic parenchyma. Two parameters were derived from the CT values measurements: 1) contrast-to-noise ratio (CNR) at different energy levels (40-140 keV, interval $10 \mathrm{keV}$ ), calculated as CNR $=\left(\mathrm{CT}_{\text {lesion }}-\mathrm{CT}_{\text {liver }}\right) / \mathrm{SD}$ noise, where $\mathrm{CT}_{\text {lesion }}$ and $\mathrm{CT}_{\text {liver }}$ are the CT values in the lesions and in the normal hepatic parenchyma and SD noise is the standard deviation for CT values of the normal parenchyma as background; 2) The lesion-to-normal parenchyma ratio (LNR), calculated as $\mathrm{LNR}=\mathrm{CT}_{\text {lesion }} / \mathrm{CT}$ liver , where $\mathrm{CT}_{\text {lesion }}$ and $\mathrm{CT}_{\text {liver }}$ are the $\mathrm{CT}$ values in the lesions and in the normal hepatic parenchyma.

Spectral CT images including conventional polychromatic images, monochromatic images and iodinebased material decomposition images were reviewed with GSI viewer in consensus at a workstation. The readers were blinded to MRI diagnosis of lesion, patient information and results of correlative imaging examinations. They recorded the following lesion features: number, density, presence of feeding vessels and enhancement pattern. To detect ability of each set of images, the true number of lesions in each image set was evaluated by comparing them with standard-ofreference findings. The relative density of the lesion with respect to the hepatic parenchyma was recorded as one of three grades of hypodensity, isodensity and hyperdensity. The enhancement pattern was described homogeneously or heterogeneously. The changes in enhancement degree or pattern between the two phases were characterized as expansion, washout or none. Finally, readers in consensus characterized each lesion as a residual HCC based on imaging findings. The typical enhancement pattern for residual HCC consisted of a rapid enhancement in the AP with a relatively quick washout in the PP, which was called "quick in and quick out". Differences between the observers were resolved by consensus conference. The sensitivity and specificity of residual HCC on the individual phase were evaluated compared to MRI consequences.

\subsection{Statistical Analysis}

Data was analyzed using SPSS ver. 19 (IBM SPSS Statistics for Windows, IBM Corp., Armonk, NY, USA). Quantitative values were recorded as mean \pm standard deviation. Receiver operating characteristic (ROC) curves were generated to help establish the threshold values of LNR required for significant differentiation of HCC. The diagnostic capability was determined by calculating the area under the ROC curve. The null hypothesis test was that the area under the ROC curve was 0.5; the alternative was that this area was greater than 0.5 .

\section{Results}

There were 30 residual lesions detected in MRI. Table 1 shows that they were found in iodine-based images and monochromatic images versus 29 in conventional CT images. The lesion-liver CNR for residual HCC at different energy levels (40-140 keV, interval $10 \mathrm{keV}$ ) showed certain regularity (Figure 1). In general, the lesion-liver CNRs at low energy levels (40-70 keV) were higher than those at high energy levels (80-140 keV). The optimal CNR for HCC could be obtained at $40 \mathrm{keV}$. Box plots of LNR in diagnosing residual HCC with conventional CT images, monochromatic images and iodine-based material decomposition images are shown in Figure 2. The results of iodine-based material decomposition images were significantly higher than the other two groups. Moreover, the ROC curves of them for detecting residual HCC are shown in Figure 3. The areas for the LNR during the AP of conventional CT, monochromatic images and iodine-based material decomposition images were 0.817 (0.677-0.956), 0.833 (0.700-0.967) and 0.933 (0.844-1.023), respectively (Table 2 ). The area of iodine-based images was evidently greater than that for other quantitative parameters for diagnosing HCC. Figure 4 shows that a residual HCC was easily missed on conventional CT images, but could be detected on iodine-based material decomposition images and $40 \mathrm{keV}$ of monochromatic images. In fact, all of the residual HCCs were consequently detected on $40 \mathrm{keV}$ of monochromatic images, especially on iodine-based material decomposition images. Therefore, the iodine-based material decomposition images showed a higher detectability than conventional polychromatic images. Figure 4 shows that monochromatic images and iodine-based images detected a hypovascular residual cancer, but conventional CT images could not detect it. Figure 5 displays that conventional CT images and monochromatic images found a false positive lesion (lipiodol deposition) as there was non-enhancement of MRI on the AP; however, 
XuY et al.

iodine-based images made a right diagnosis due to a still hyperattenuated lesion on the PP. Figure 6 shows that iodine-based material decomposition images and $40 \mathrm{keV}$ of monochromatic images clearly displayed the residual HCCs and spectral CT contained many methods to demonstrate the LNR of residual HCCs.

Table 1. Number of True Positive, False Positive and False Negative of Images

\begin{tabular}{lccc}
\hline Images & True Positive & False Positive & False Negative \\
\hline Iodine-based images & 30 & 0 & 0 \\
Monochromatic images & 30 & 1 & 0 \\
Conventional CT images & 29 & 1 & 1 \\
\hline
\end{tabular}

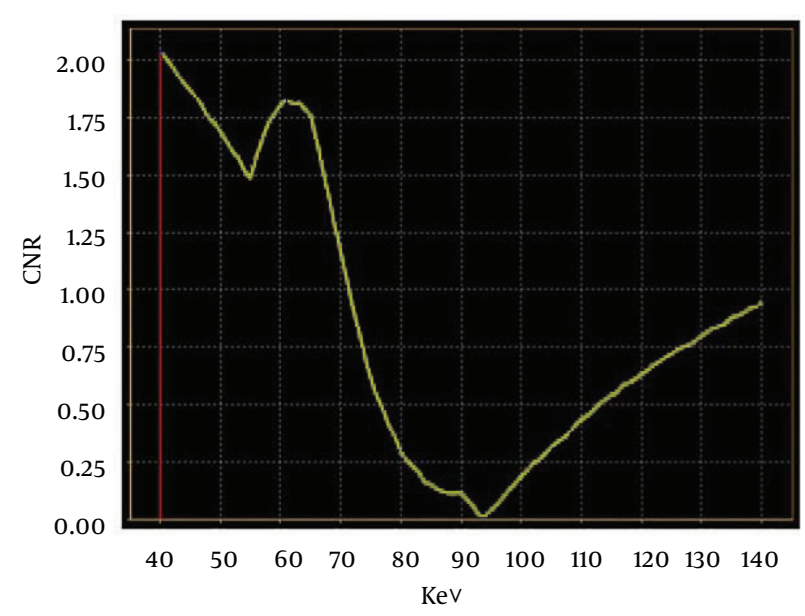

Figure 1. CNR for residual HCC at different energy levels (40-140 keV, Interval $10 \mathrm{keV}$ ) on the AP. CNR values peaked at $40 \mathrm{keV}$.

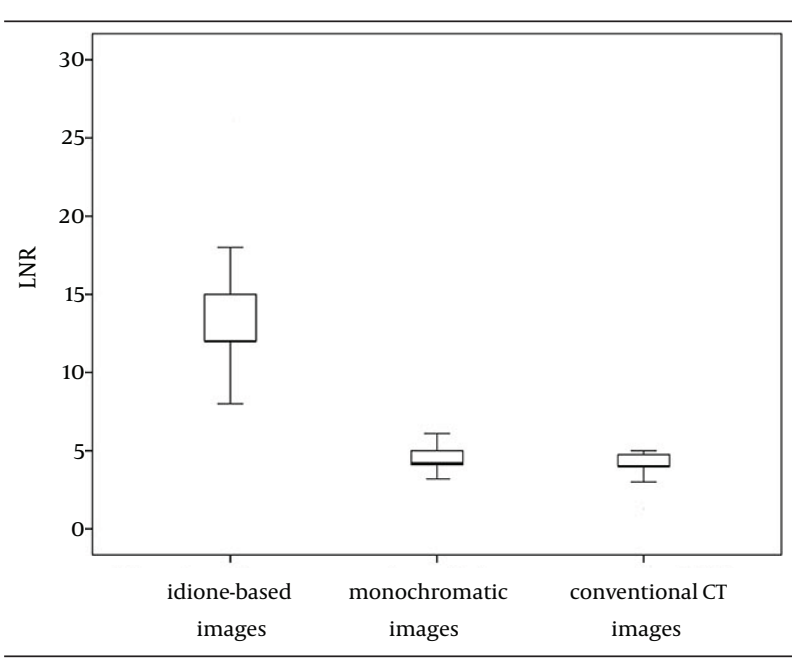

Figure 2. Box plots of LNR in diagnosing residual HCC with conventional CT images, monochromatic images and iodine-based material decomposition images. The average LNR of iodine-based material decomposition images was greater than that of monochromatic images and the average LNR of monochromatic images was greater than that of conventional CT images.

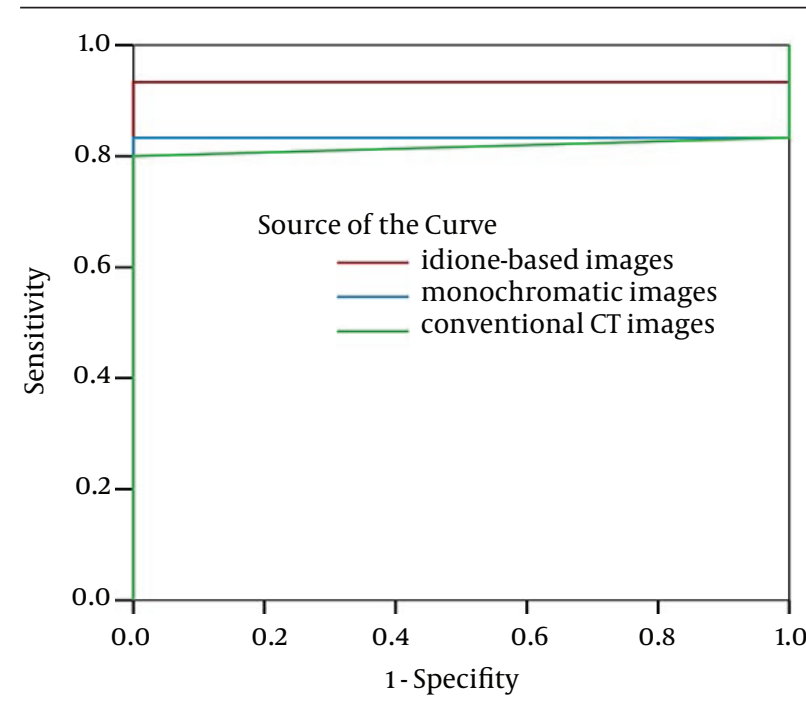

Figure 3. ROC curves for LNR on the AP in diagnosing residual HCC with conventional CT images, monochromatic images and iodine-based images. The area under ROC curve of iodine-based material decomposition images was greater than that of monochromatic images and the area under the ROC curve of monochromatic images was greater than that of conventional CT images.

Table 2. Areas Under the ROC Curves for Detecting Residual $\mathrm{HCC}^{\mathrm{a}}$

\begin{tabular}{lccc}
\hline Images & Area & $\begin{array}{c}\text { Standard } \\
\text { Error }\end{array}$ & $\begin{array}{c}\text { 95\% Confidence } \\
\text { Interval }\end{array}$ \\
\hline $\begin{array}{l}\text { Iodine-based } \\
\text { images }\end{array}$ & 0.933 & 0.046 & $0.844-1.023$ \\
$\begin{array}{l}\text { Monochromatic } \\
\text { images }\end{array}$ & 0.833 & 0.068 & $0.700-0.967$ \\
$\begin{array}{l}\text { Conventional CT } \\
\text { images }\end{array}$ & 0.817 & 0.071 & $0.677-0.956$ \\
\hline
\end{tabular}

a Abbreviations: HCC; Hepatocellular Carcinoma, ROC; Receiver Operating Characteristic. 

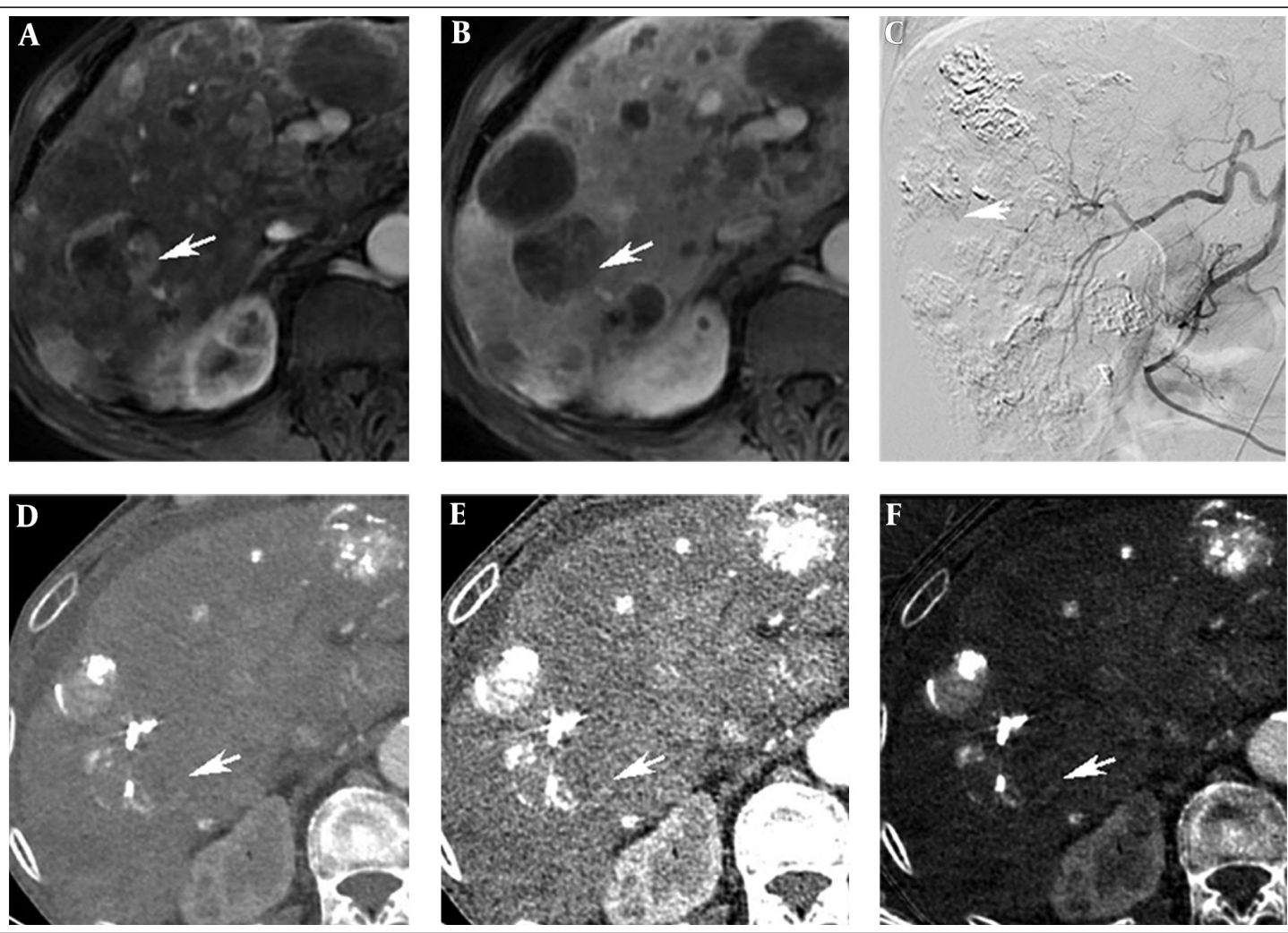

Figure 4. A 71-year-old man with a 42-mm HCC in the right posterior lobe of liver A A Residual cancer in MRI on the AP (arrow). B, Residual cancer in MRI on the PP (arrow).C, Interventional CT image showed a hyperattenuated lesion (arrow). D, Conventional polychromatic image on the AP did not show the lesion clearly. E, Monochromatic image at $40 \mathrm{keV}$ on the AP. F, Iodine-based material decomposition image on the AP showed a hyperattenuated lesion (arrow).
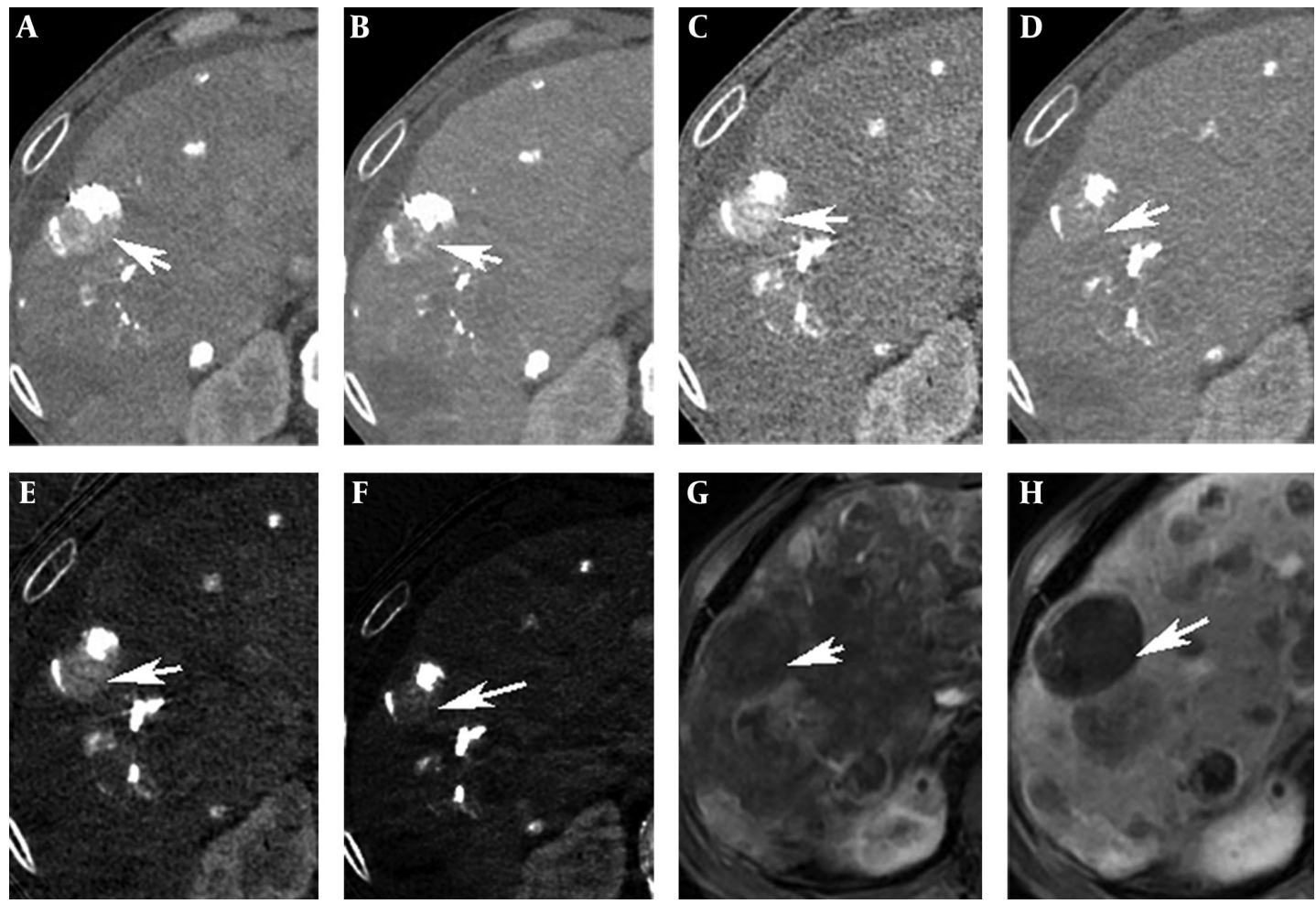

Figure 5. A 71-year-old man with a 42-mm HCC in the right posterior lobe of liver. A and B, Conventional CT image on the AP and PP. C and D, Monochromatic image at $40 \mathrm{keV}$ on the AP and PP showed a likely residual cancer (arrows). E and F, Iodine-based material decomposition image on the AP and PP showed a still hyperattenuated lesion on the PP (arrows). G, H, MRI on the AP and PP presented no residual cancer (arrows). 

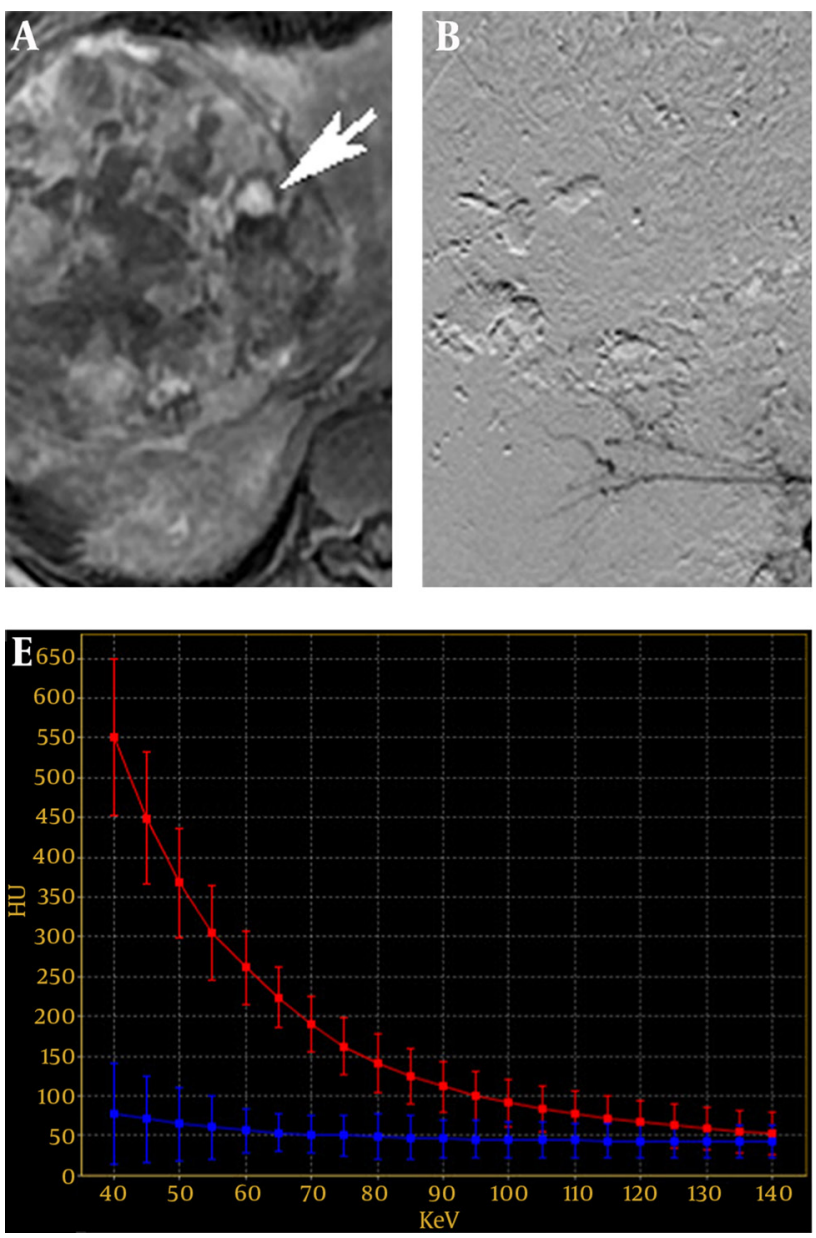
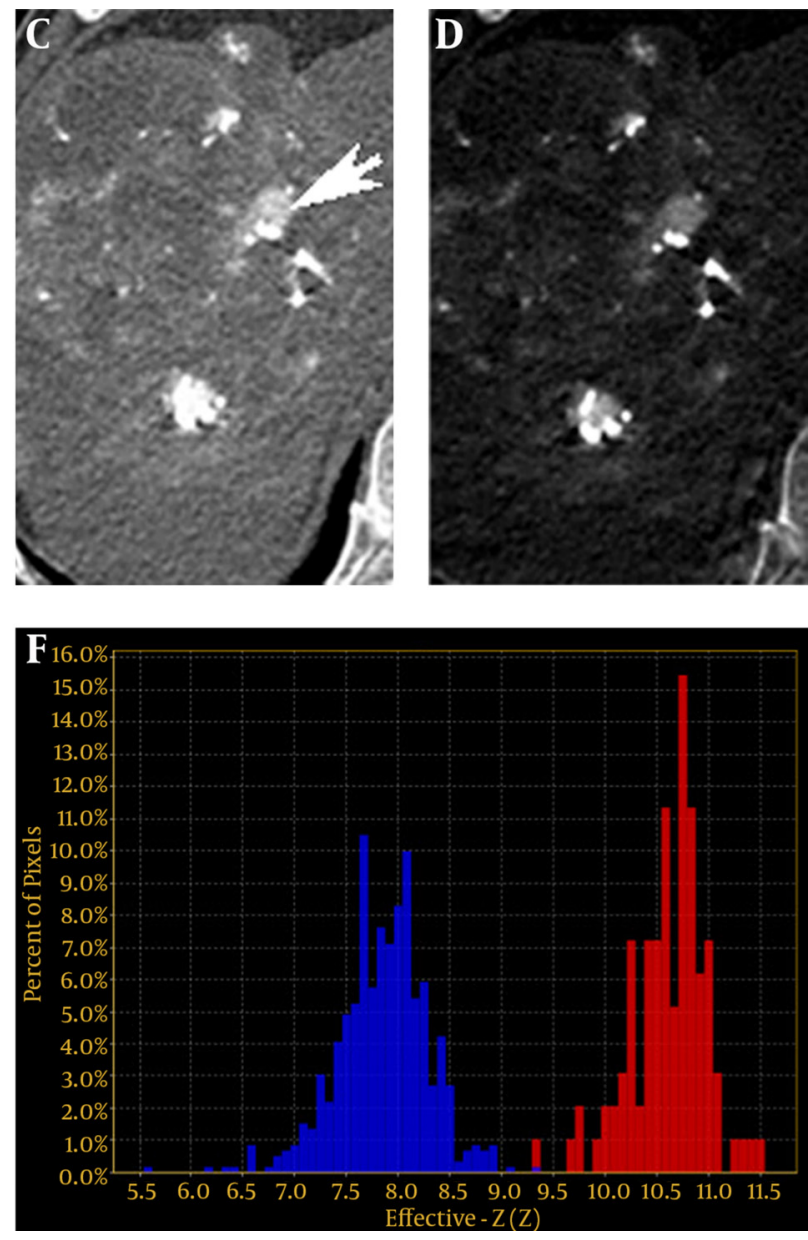

Figure 6. A 51-year-old man with a 140-mm HCC in the right lobe of liver. A, A residual cancer of MRI on the AP (arrow). B, Interventional image showed no residual cancer. C, Monochromatic image at $40 \mathrm{keV}$ on the AP showed a hyperattenuated lesion (arrow). D, Iodine-based material decomposition image on the AP. E and F, Spectral curves and histogram for residual cancer and liver were obviously different (red is the residual HCC, blue is the liver parenchyma).

\section{Discussion}

HCC is the sixth most frequent cancer worldwide and the third most prevalent cancer resulting in death (15). However, only a few patients with HCCs are surgical candidates at diagnosis $(16,17)$. TACE is one of the most ordinarily performed therapies to treat unresectable HCCs. TACE usually contains intra-arterial delivery of emulsions mixed with chemotherapeutic agents and lipiodol, followed by administration of embolic agent. It has been demonstrated that the amount of intratumoral lipiodol deposition and the proportion of residual cancer associate well to tumor necrosis, tumor recurrence and survival rate after TACE (18-20). Accurate and quick assessment of lipiodol deposition and residual cancer is especially relevant for HCC after TACE and provides interventional radiologist with better information to evaluate the effect of TACE and to offer feedback for TACE (18-22). MRI is optimal to detect viable tumor with higher sensitivity owing to better contrast of different soft tissues and higher spatial resolution than CT; whereas, absence of presentation of lipiodol disposition. In clinical practice, CT is usually performed before MRI due to its cost-effectiveness. Although CT is usually used to monitor lipiodol disposition of TACE for HCC, there is a considerable difficulty in accurately detecting residual viable tumor with this modality, because high attenuation of iodized oil makes it difficult to differentiate areas of true enhancement from areas of reserved lipiodol. For example, in our study, the conventional CT images omitted a positive residual cancer. In this study, spectral CT imaging not only contained the monochromatic CT images and iodine-based material decomposition images for the detection and characterization of residual cancer, but also provided kinds of quantitative maps (e.g. spectral curve, scatterplot, histogram) to distinguish residual cancer from adjacent liver. Spectral CT imaging can produce monochromatic images at energy levels ranging from 40 to $140 \mathrm{keV}$, 
which is devoid of averaging attenuation effects (23). This would promote increasing contrast spatial resolution. The optimal monochromatic images obtained at optimal $\mathrm{keV}$ markedly improved CNR of residual HCC. As shown in our study, $40 \mathrm{keV}$ monochromatic images showed a higher ability to detect HCCs than conventional polychromatic images. The above omitted residual cancer by conventional CT was detected in spectral CT. Iodine-based material decomposition images were used for quantifying iodine concentrations whatever the amount of iodine (24). For hypovascular liver lesions, iodine-based material decomposition images could show focal uptake of iodinated contrast material obviously attributed to low defect of lipiodol deposition. For example, there was a lipiodol deposition mistaken for a false residual in the conventional CT images and monochromatic images; whereas, the iodine-based material decomposition images provided a right diagnosis. Besides, as shown in our study, the average LNR of iodine-based material decomposition images was significantly higher than the conventional CT images, which showed a higher ability of displaying residual HCCs. ROC curves analyses in the present study revealed that compared with conventional qualitative image analysis, iodine-based material decomposition images with spectral CT improved the sensitivity. The iodine concentration lesions derived from the iodine-based material decomposition images are quantitative, as demonstrated in in vitro experiments, and thus might be a useful parameter (23).

The present study had some limitations. First, the results were preliminary and need to be verified by additional studies on larger number of lesions. Second, because the readers assessed the images in consensus, intra- or interobserver variability data are lacking. In conclusion, spectral CT imaging with quantitative analysis of iodine concentration may be helpful for increasing the accuracy of follow-up of patients with HCC after TACE. Further research is necessary to evaluate clinical value of quantitative measurements of iodine concentrations.

\section{Acknowledgements}

None declared.

\section{Authors' Contributions}

Study concept and design: Guixiang Zhang. Analysis and interpretation of data: Yanhong Xu. Drafting of the manuscript: Yanhong Xu. Critical revision of the manuscript for important intellectual content: An Xiao and Jia Yang. Statistical analysis: Zaixian Zhang.

\section{References}

1. El-Serag HB. Hepatocellular carcinoma. N Engl J Med. 2011; 365(12):1118-27.

2. Jemal A, Bray F, Center MM, Ferlay J, Ward E, Forman D. Global cancer statistics. CA Cancer J Clin. 2011;61(2):69-90.

3. Maluccio M, Covey A. Recent progress in understanding, diag- nosing, and treating hepatocellular carcinoma. CA Cancer J Clin. 2012;62(6):394-9.

4. Miao R, Luo H, Zhou H, Li G, Bu D, Yang X, et al. Identification of prognostic biomarkers in hepatitis B virus-related hepatocellular carcinoma and stratification by integrative multi-omics analysis. J Hepatol. 2014;61(4):840-9.

5. Altekruse SF, McGlynn KA, Reichman ME. Hepatocellular carcinoma incidence, mortality, and survival trends in the United States from 1975 to 2005.J Clin Oncol. 2009;27(9):1485-91.

6. Kim do Y, Han KH. Epidemiology and surveillance of hepatocellular carcinoma. Liver Cancer. 2012;1(1):2-14.

7. Yeganeh B, Hashemi M, de Serres FJ, Los MJ, Ghavami S. Different faces of hepatocellular carcinoma as a health threat in 21st century. Hepat Mon. 2013;13(2):e9308.

8. Sherman M. Hepatocellular carcinoma: epidemiology, surveillance, and diagnosis. Semin Liver Dis. 2010;30(1):3-16.

9. Mazioti A, Gatselis NK, Rountas C, Zachou K, Filippiadis DK, Tepetes $K$, et al. Safety and efficacy of transcatheter arterial chemoemboliazation in the real-life management of unresectable hepatocellular carcinoma. Hepat Mon. 2013;13(8):e7070.

10. Ghanaati H, Alavian SM, Jafarian A, Ebrahimi Daryani N, NassiriToosi M, Jalali AH, et al. Imaging and Imaging-Guided Interventions in the Diagnosis and Management of Hepatocellular Carcinoma (HCC)-Review of Evidence. Iran J Radiol. 2012;9(4):167-77.

11. Kubota K, Hisa N, Nishikawa T, Fujiwara Y, Murata Y, Itoh S, et al. Evaluation of hepatocellular carcinoma after treatment with transcatheter arterial chemoembolization: comparison of Lipiodol-CT, power Doppler sonography, and dynamic MRI. Abdom Imaging. 2001;26(2):184-90.

12. Chen CY, Li CW, Kuo YT, Jaw TS, Wu DK, Jao JC, et al. Early response of hepatocellular carcinoma to transcatheter arterial chemoembolization: choline levels and MR diffusion constants-initial experience. Radiology. 2006;239(2):448-56.

13. Kubota K, Yamanishi T, Itoh S, Murata Y, Miyatake K, Yasunami $\mathrm{H}$ et al. Role of diffusion-weighted imaging in evaluating therapeutic efficacy after transcatheter arterial chemoembolization for hepatocellular carcinoma. Oncol Rep. 2010;24(3):727-32.

14. Huppertz A, Balzer T, Blakeborough A, Breuer J, Giovagnoni A, Heinz-Peer G, et al. Improved detection of focal liver lesions at MR imaging: multicenter comparison of gadoxetic acidenhanced MR images with intraoperative findings. Radiology. 2004;230(1):266-75.

15. Wang Z, Lin M, Lesage D, Chen R, Chapiro J, Gu T, et al. Threedimensional evaluation of lipiodol retention in HCC after chemoembolization: a quantitative comparison between CBCT and MDCT. Acad Radiol. 2014;21(3):393-9.

16. Forner A, Llovet JM, Bruix J. Hepatocellular carcinoma. Lancet. 2012;379(9822):1245-55.

17. Marin-Hargreaves G, Azoulay D, Bismuth H. Hepatocellular carcinoma: surgical indications and results. Crit Rev Oncol Hematol. 2003;47(1):13-27.

18. Monsky WL, Kim I, Loh S, Li CS, Greasby TA, Deutsch LS, et al. Semiautomated segmentation for volumetric analysis of intratumoral ethiodol uptake and subsequent tumor necrosis after chemoembolization. AJR Am J Roentgenol. 2010;195(5):1220-30.

19. Takayasu K, Muramatsu Y, Maeda T, Iwata R, Furukawa H, Muramatsu Y, et al. Targeted transarterial oily chemoembolization for small foci of hepatocellular carcinoma using a unified helical CT and angiography system: analysis of factors affecting local recurrence and survival rates. AJR Am J Roentgenol. 2001;176(3):681-8.

20. Lee HS, Kim KM, Yoon JH, Lee TR, Suh KS, Lee KU, et al. Therapeutic efficacy of transcatheter arterial chemoembolization as compared with hepatic resection in hepatocellular carcinoma patients with compensated liver function in a hepatitis B virus-endemic area: a prospective cohort study. J Clin Oncol. 2002;20(22):4459-65.

21. Takayasu K, Arii S, Matsuo N, Yoshikawa M, Ryu M, Takasaki K, et al. Comparison of CT findings with resected specimens after chemoembolization with iodized oil for hepatocellular carcinoma. AJR Am J Roentgenol. 2000;175(3):699-704.

22. Lin XZ, Wu ZY, Tao R, Guo Y, Li JY, Zhang J, et al. Dual energy spectral CT imaging of insulinoma-Value in preoperative diagnosis compared with conventional multi-detector CT. Eur J Radiol. 
2012;81(10):2487-94

23. Lv P, Lin XZ, Li J, Li W, Chen K. Differentiation of small hepatic hemangioma from small hepatocellular carcinoma: recently introduced spectral CT method. Radiology. 2011;259(3):720-9.
24. Zhang XF, Lu Q, Wu LM, Zou AH, Hua XL, Xu JR. Quantitative iodine-based material decomposition images with spectral CT imaging for differentiating prostatic carcinoma from benign prostatic hyperplasia. Acad Radiol. 2013;20(8):947-56. 